\title{
Acetyl-coenzyme a carboxylase - an attractive enzyme for biotechnology
}

\begin{abstract}
Acetyl-coenzyme A carboxylase is a key enzyme initiating the synthesis of biologically active compounds, fixation of inorganic carbon $\left(\mathrm{CO}_{2}\right)$, fuel precursor production, and the production of novel antibiotics. The enzyme can catalyse biological transformations for effective production of bioactive metabolites
\end{abstract}

Keywords: acetyl-coenzyme, active compounds; $\mathrm{CO}_{2}$, antibiotics, biomolecules
Volume 7 Issue | - 2018

\author{
Ugoala Emeka \\ National Institute for Freshwater Fisheries Research, P. M. B \\ 6006, New Bussa, Niger State, Nigeria
}

Correspondence: Ugoala Emeka, National Institute for Freshwater Fisheries Research, P. M. B 6006, New Bussa, Niger State, Nigeria, Email nnaemekaugoala@gmail.com
Abbreviations: TMOF, trypsin-modulating oostatic factor; ACC, acetyl-CoA carboxylase; T2DM, type 2 diabetes mellitus; ACCase, acetyl CoA carboxylase

\section{Introduction}

The current demand in modern medicine and nutrition have necessitated the exploration of biological systems for biomolecules making bio-prospecting a very great present interest. However, advances in biotechnology, particularly molecular farming (molecular pharming, biopharming or gene pharming) generates biomolecules valuable to medicine or industry that are difficult or even impossible to produce in another way, or which require prohibitively high production costs in other systems. These bio-chemicals (secondary metabolites) considered as specialty materials or fine chemicals, are used commercially as biologically active compounds (pharmaceuticals, flavours, fragrances, and pesticides) or as drug entities or as model compounds for drug syntheses and semi-syntheses. ${ }^{1,2}$

Naturally, secondary metabolites are accumulated in smaller quantities, limited in distribution in containing organisms (restricted to a particular species, genus, family, or closely related group of families), synthesized in specialized cell types and at distinct developmental stages, making their extraction and purification difficult. Acetylcoenzyme A carboxylase is responsible for carboxylation of acetyl$\mathrm{CoA}$ that results in malonyl-coenzyme A formation. Malonyl-CoA is a major building block for compounds such as fatty acids, polyketides and flavonoids - important components for cell growth, as well as for food, pharmaceuticals and energy production. ${ }^{3}$ It controls carbon flux to fatty acid biosynthesis and from $\beta$-oxidation allows for the biosynthesis of natural products of biotechnological importance. The recent emergence of biotechnological manipulation can be targeted to modulate acetyl-coenzyme A carboxylase activity in bacteria, plants and animals ${ }^{4,5}$ to provide the platforms for effective production of bioactive metabolites as a feasible alternative to biochemical screening schemes.

\section{Antibody and vaccine production}

Antibody and vaccine production are more convenient and much cheaper in organism without an immune system. Successful expression and assembly of a recombinant human monoclonal IgA antibody has already been demonstrated for Chlamydomonas reinhardtii, ${ }^{6}$ while stable expression of the hepatitis B surface antigen gene has been shown in Dunaliella salina. ${ }^{7}$ Since Dunaliellais otherwise used for nutrition, there is no need for purification of the antigen, so the intact algae could be used to deliver a vaccine.

Microalgae is also useful for expressing insecticidal proteins. Because the green alga Chlorella is one possible food for mosquito larvae, the mosquito hormone trypsin-modulating oostatic factor (TMOF) was heterologously expressed in Chlorella. TMOF causes termination of trypsin biosynthesis in the mosquito gut. After feeding mosquito larvae with these recombinant Chlorellacells the larvae died within $72 \mathrm{~h} .{ }^{8}$ Mosquito abatement is an expensive requirement in tropical countries which the use of transgenic algae might be a much cheaper alternative.

\section{Male infertility reversal and hybrid seed breeding}

Acetyl-CoA is the major component involved in the biosynthesis of fatty acid which results into male fertility. Fertility restorer gene $\mathrm{R}$ is dominant in nature and found in certain strains of same species or can be transferred from the related species.

\section{Treatment of obesity and diabetes (metabolic syndrome)}

The development of acetyl-CoA carboxylase (ACC) inhibitors for the treatment of metabolic disease is presently being pursued by the pharmaceutical industry. Acetyl-CoA carboxylase (ACC) inhibitors offer significant potential for the treatment of type 2 diabetes mellitus (T2DM), hepatic steatosis, and cancer.

Humans have two forms of ACC. One, expressed in the cytoplasm of liver and fat cells, is essential for fatty acid synthesis. The other form is expressed in muscle cells and is transported into mitochondria, where it plays a role in the regulation of fatty acid oxidation. The second form inhibition controls weight gain. Deficient in acetyl-coenzyme A carboxylase would necessitate elevated fatty acid oxidation and reduced body fat content and body weight, even if more food are consumed. Therefore, inhibitors against ACCs might be efficacious for the treatment of obesity and diabetes (metabolic syndrome).

\section{Production of polyphenols from microorganisms}

Polyphenols as antioxidants can combat cardiovascular disease, certain types of cancer, neurodegenerative diseases, allergies, diabetes and inflammation. A small genome and rapid growth characteristics, 
makes Lactococcus lactis an ideal host for production of valuable food and pharmaceutical compounds. But malonyl-CoA availability is a bottleneck in its utilization. The malonyl-CoA levels can be modulated by acetyl-CoA carboxylase activity in the engineered strains.

\section{$\mathrm{CO}_{2}$ fixation in plants}

Atmospheric $\mathrm{CO}_{2}$ is a cheap and readily available carbon source that can be used to synthesize useful bio-chemicals. However, uncatalyzed chemical $\mathrm{CO}_{2}$-fixation reactions usually require quite harsh conditions to functionalize the $\mathrm{CO}_{2}$ molecule. Nature provides a multitude of different carboxylating enzymes whose carboxylating principle(s) might be exploited in biotechnology. Acetyl-CoA carboxylase can catalyze autotrophic carbon dioxide fixation in a thermophilic and acidophilic archeon. ${ }^{9}$

\section{Biofuel production}

Acetyl CoA Carboxylase (ACCase) can be used to catalyze a key metabolic step in the synthesis of oils in algae. Acetyl-CoA carboxylase (ACC) catalyzes the biotin-dependent carboxylation of acetyl-CoA to produce malonyl-CoA. This is the first and the committed step in the biosynthesis of long-chain fatty acids. ${ }^{10}$

\section{Acknowledgements}

None.

\section{Conflicts of interest}

I have no conflicts of interest to declare.

\section{References}

1. Bingel AS. New Natural Products and Plant Drugs with Pharmacological, Biological or Therapeutical Activity. Wagner H \& Wolff P (Eds.) Springer-Verlan, New York, USA, 1977. p. 1-22.
2. Roche EB. Design of Biopharmaceutical Properties through Prodrugs and Analogs American Pharmaceutical Association Academy of Pharmaceutical Sciences, Washington, USA. 1977.

3. Podkowiński Jan, Tworak Aleksander. Acetyl-coenzyme A carboxylasean attractive enzyme for biotechnology. Journal of Biotechnology, Computational Biology and Bionanotechnology. 2011;92(4):321-335.

4. Brigham CJ, Budde CF, Holder JW, et al. Elucidation of beta-oxidation pathways in RalstoniaeutrophaH16 by examination of global gene expression. J Bacteriol. 2011;192(20):5454-5464.

5. Brigham CJ, Speth DR, Rha C, et al. Whole genome microarray and gene deletion studies reveal regulation of the polyhydroxyalkanoate production cycle by the stringent response in Ralstonia eutrophaH16. Appl Environ Microbiol. 2012;78(22):8033-8044.

6. Mayfield SP, Franklin SE, Lerner RA. Expression and assembly of a fully active antibody in algae. Proceedings of the National Academy of Sciences USA. 2003;100(2):438-442.

7. Geng D, Wang Y, Wang P, et al. Stable expression of hepatitis B surface antigen gene in Dunaliella salina (Chlorophyta). Journal of Applied Phycology. 2003;15(6):451-456.

8. Borovsky D.Trypsin-modulating oostatic factor: a potential new larvicide for mosquito control. J Exp Biol. 2003;206(21):3869-3875.

9. Ishii M, Chuakrut S, Arai H, Igarashi Y. Occurrence, biochemistry and possible biotechnological application of the 3-hydroxypropionate cycle. Appl Microbiol Biotechnol. 2004;64(5):605-610.

10. Siripornadulsil S, Traina S, Verma DP, et al. Molecular mechanisms of proline-mediated tolerance to toxic heavy metals in transgenic microalgae. Plant Cell. 2002;14(11):2837-2847. 